\title{
Pricing Loan CDS with Vasicek Interest Rate under the Contagious Model
}

\author{
Yinglin Liu1, Ruili Hao²*, Zuhua Wang ${ }^{3}$ \\ ${ }^{1}$ Faculty of Business and Economics, Macquarie University, Sydney, Australia \\ ${ }^{2}$ Department of Financial Mathematics, Shanghai Finance University, Shanghai, China \\ ${ }^{3}$ School of Mathematics, Shanghai University of Finance and Economics, Shanghai, China \\ Email: *haoruili13@163.com
}

Received 20 May 2016; accepted 23 August 2016; published 26 August 2016

Copyright (C) 2016 by authors and Scientific Research Publishing Inc.

This work is licensed under the Creative Commons Attribution International License (CC BY). http://creativecommons.org/licenses/by/4.0/

(c) (i) Open Access

\section{Abstract}

This paper mainly studies the pricing of credit default swap with the loan as the reference asset under the primary-secondary model. In the contract of credit default swap (CDS), we consider that the defaults of the counterparties are correlated with the stochastic interest rate following Vasicek model or the default state of the reference firm. We assume that the company's default is independent with the company's prepayment and obtain the pricing formulas of the loan and loan CDS.

\section{Keywords}

\section{Loan CDS, Contagious Risk, Vasicek Interest Rate, Primary-Secondary Framework}

\section{Introduction}

Since the end of the twentieth century, the derivatives market has developed rapidly and become one of the most important financial innovations in the internationally financial market. It has also become a new tool of managing credit risk after the loan transaction and the asset securitization. Credit default swap (CDS) is one of the most important derivatives to manage credit risk in the financial market.

Because it is easy to implement standardization, the credit default swap market has the rapid expansion. However, some concealed contradictions exposed gradually, such as the United States subprime crisis and the European sovereign debt crisis. They make people realize that credit derivatives bring the convenience and contain huge risk at the same time, especially contagious risk. Therefore, the pricing problem of credit default swap became a hot research topic in recent years.

${ }^{*}$ Corresponding author. 
Until now, there have been mainly two basic approaches to study credit risk: the structural approach and the reduced approach. The corresponding models are called the structural model and the reduced-form model. The structural approach introduced the firm's default governed by the value of its assets and debts such as [1] [2]. However, for the problem of the valuation of credit products with jump-diffusion risk, it is still difficult to get explicit results, even using the above approaches in the event of defaulting before the maturity date. Nevertheless, the reduced-form approach is comparatively flexible and tractable to solve this problem. For the reduced-form approach, exogenous mechanism of firm's default was introduced. This model considers the default as a random event which was controlled by an exogenous intensity process (see [3]-[5]). With the more aggregate credit risk in the modern financial markets, we have recognized that the defaults of many firms have direct linkage. Thereby the valuation of credit securities with contagious risk has aroused a lot of authors' interests. The model of credit contagion was firstly proposed to account for the concentration risk in large portfolios of defaultable securities (DL Model) in [6]. Later, DL Model was generalized and the concept of counterparty risk which was from the default of firm's counterparties was firstly introduced in [7]. Because it is impossible to assume that the impact of one firm's default to another firm's default keeps constant all the time, some authors introduced a hyperbolic function to reflect the attenuation effect in [8]. Recently, the cases that the interest rate satisfied the jump-diffusion process and the fractional Brownian motion were also discussed in [9]-[11]. The above conclusions on CDS were mostly obtained when the reference assets were the bonds.

With the rapid development of the financial securities and financial derivatives, the proportion of the financial assets in the total assets of the society is increasing. Therefore, the ability and the level of managing the risk for the financial institutions have become the decisive factors to improve their competitiveness and profitability. As a special enterprise, bank has a special role in the economical development. Its main business is to deposit and provide the loans. The credit risk of the bank is mainly derived from the loans. In China, credit risk is excessively concentrated. The existing methods and tools managing credit risk are ineffective. Thus, making use of domestic and foreign research on credit derivatives, the exploration and the development of credit derivatives to transfer credit risk in China's market become very necessary. However, most conclusions on credit derivatives based on the loan were qualitative and there are few deeply quantitative research. [12] studied the pricing of mortgage CDS under structured model. [13] considered the characteristics in various types of loans. They proposed a new idea of using CDS to transfer their risks and gave the pricing model of CDS. The research on credit default swap based on the loan in foreign countries mostly used the reduced method such as [14] [15]. The above studies did not consider the contagious risk among the counterparties. [16] discussed the pricing problem of loan CDS with contagious risk. But the loan was particular in [16] and it had a cash deposit. In this paper, we will make use of the contagious model with attenuation effect to study the pricing of CDS based on fully amortizing fixed-rate mortgage (FRM). This kind of the loan is very common. Therefore, the conclusions in this paper will provide the theoretical preparation and the suggestions for the credit products development and the research in China.

\section{The Structure of the Default and the Prepayment}

Suppose $\left(\Omega, \mathcal{F},\left\{\mathcal{F}_{t}\right\}_{t=0}^{T^{*}}, Q\right)$ be the filtered probability space satisfying the usual conditions. $\mathcal{F}=\mathcal{F}_{T^{*}}, T^{*}<\infty$ and $Q$ is an equivalent martingale measure under which discounted securities' prices are martingales and is unique. The point processes $\left\{M_{t}^{i}\right\}_{i=A, B}$ are the default processes of firm A and firm B and $\left\{N_{t}^{i}\right\}_{i=A, B}$ is the process of repaying the loan in advance of firm A and firm B. When $M_{t}^{i}$ firstly jumps from 0 to 1 , we call the firm $i$ defaults and denote $\tau_{d}^{i}$ be the default time of company $i$. When $N_{t}^{i}$ firstly jumps from 0 to 1 , we call the firm $i$ repays the remained loan in advance and denote $\tau_{p}^{i}$ be the time of the repayment in advance. $I_{\{\}}$is the indicator function, then $M_{t}^{i}=\mathrm{I}_{\left\{\tau_{d}^{i}<t\right\}}, N_{t}^{i}=\mathrm{I}_{\left\{\tau_{p}^{i}<t\right\}}$. We assume that the unique macro state variable is the interest rate $r=\left\{r_{t}, t \geq 0\right\}$. Denote

$$
\mathcal{F}_{t}=\mathcal{F}_{t}^{r} \vee \mathcal{F}_{t}^{A} \vee \mathcal{F}_{t}^{B} \vee \mathcal{H}_{t}^{A} \vee \mathcal{H}_{t}^{B},
$$

where

$$
\mathcal{F}_{t}^{r}=\sigma\left(r_{s}, 0<s<t\right), \mathcal{F}_{t}^{i}=\sigma\left(M_{s}^{i}, 0<s<t\right), \mathcal{H}_{t}^{i}=\sigma\left(N_{s}^{i}, 0<s<t\right)
$$


Let

$$
\begin{aligned}
& \mathcal{G}_{0}^{A}=\mathcal{F}_{T^{*}}^{B} \vee \mathcal{F}_{T^{*}}^{r}, \mathcal{G}_{0}^{B}=\mathcal{F}_{T^{*}}^{A} \vee \mathcal{F}_{T^{*}}^{r}, \\
& \mathcal{G}_{t}^{i}=\mathcal{F}_{t} \vee \mathcal{G}_{0}^{i} .
\end{aligned}
$$

Suppose that $\tau_{d}^{i}(i=A, B)$ and $\tau_{p}^{i}(i=A, B)$ respectively have the positively $\mathcal{G}_{0}^{i}$-measurable processes $\lambda_{t}^{i}$ and $\xi_{t}^{i}$. For $t \in\left[0, T^{*}\right], \int_{0}^{t} \lambda_{s}^{i} \mathrm{~d} s<\infty$ and $\int_{0}^{t} \xi_{s}^{i} \mathrm{~d} s<\infty, P-a . s$.

We can define $\tau_{d}^{i}$ and $\tau_{p}^{i}$ as following

$$
\tau_{d}^{i}=\inf \left\{t: \int_{0}^{t} \lambda_{s}^{i} \mathrm{~d} s \geq E^{i}\right\}, \quad \tau_{p}^{i}=\inf \left\{t: \int_{0}^{t} \xi_{s}^{i} \mathrm{~d} s \geq E^{j}\right\},
$$

where $\left\{E^{i}, 0<i<n\right\},\left\{E^{j}, 0<j<n\right\}$ are independent. The conditional and unconditional distributions of $\tau_{d}^{i}$ and $\tau_{p}^{i}$ are given by

$$
\begin{array}{ll}
P\left(\tau_{d}^{i}>t \mid \mathcal{G}_{0}^{i}\right)=\exp \left(-\int_{0}^{t} \lambda_{u}^{i} \mathrm{~d} u\right), t \in\left[0, T^{*}\right], & P\left(\tau_{d}^{i}>t\right)=E\left[\exp \left(-\int_{0}^{t} \lambda_{u}^{i} \mathrm{~d} u\right)\right], t \in\left[0, T^{*}\right], \\
P\left(\tau_{p}^{i}>t \mid \mathcal{G}_{0}^{i}\right)=\exp \left(-\int_{0}^{t} \xi_{u}^{i} \mathrm{~d} u\right), t \in\left[0, T^{*}\right], & P\left(\tau_{p}^{i}>t\right)=E\left[\exp \left(-\int_{0}^{t} \xi_{u}^{i} \mathrm{~d} u\right)\right], t \in\left[0, T^{*}\right] .
\end{array}
$$

The joint distributions of $\tau_{d}^{i}$ and $\tau_{p}^{i}$ are given by

$$
\begin{aligned}
& P\left(\tau^{i}>t \mid \mathcal{G}_{0}^{i}\right)=P\left(\tau_{d}^{i} \wedge \tau_{p}^{i}>t \mid \mathcal{G}_{0}^{i}\right)=\exp \left[-\left(\int_{0}^{t} \lambda_{u}^{i} \mathrm{~d} u+\int_{0}^{t} \xi_{u}^{i} \mathrm{~d} u\right)\right], \quad t \in\left[0, T^{*}\right], \\
& P\left(\tau^{i}>t\right)=E\left\{\exp \left[-\left(\int_{0}^{t} \lambda_{u}^{i} \mathrm{~d} u+\int_{0}^{t} \xi_{u}^{i} \mathrm{~d} u\right)\right]\right\}, \quad t \in\left[0, T^{*}\right] .
\end{aligned}
$$

\section{The Pricing of the Loan}

The reference asset of credit default swap is a loan which takes the installments. After the signing of the loan contract, the borrower of the loan promises to repay equal amount of the principal and the interest to the lender in each repayment date which is called Fully Amortizing Fixed-rate Mortgage (FRM). Until the maturity date, the borrowers should repay all the principal and the interest. In addition, the lender generally requires the borrower to issue a corresponding collateral before the contract in order to improve the borrower's credit rating and the credit limits. The collateral can be a basket of the financial assets or some physical assets. In this paper, we assume that the repayment of the loan satisfies two conditions: 1) The time of the payment is continuous; 2) Allow the borrower to repay the loan in advance, but need repay all the loans once time.

The borrower may choose to repay the loan in advance, default or hold a loan. We assume that $L$ is the repayment amount of the borrower in the unit time. $M(t)$ is the remaining loan at time $t . T$ is the maturity date of the loan. $\tau_{d}$ and $\tau_{p}$ are respectively the default time of company $i$ and the time of the repayment in advance. $R(t)$ is the recovery rate of the loan when the borrower defaults. $L, M(t)$ and $R(t)$ are not stochastic. $\tau=\min \left(\tau_{d}, \tau_{p}\right)$ denotes the stop time of repayment for the loan. If $\tau=\tau_{p}$, the remained loan is $M\left(\tau_{p}\right)$. If $\tau=\tau_{d}$, the remained loan is $M\left(\tau_{d}\right)$ and the bank can recover $M\left(\tau_{d}\right) R\left(\tau_{d}\right)$. Thus, the cash flow is the continuous repayment $Y$ if the borrower dose not default or repay the loan in advance before the maturity date. In a no-arbitrage market, the value of FRM is the discount of the expectation of the future cash flow on the risk-neutral measure.

Therefore, the price of the loan is

$$
\begin{aligned}
V(t)= & E_{t}^{Q}\left[M\left(\tau_{p}\right) \mathrm{e}^{-\int_{t}^{\tau_{p}} r(u) \mathrm{d} u} I_{\left\{\tau_{p}<T\right\}}\right]+E_{t}^{Q}\left[\int_{t}^{T} L \mathrm{e}^{-\int_{t}^{s} r(u) \mathrm{d} u} I_{\left\{\tau_{p}<T\right\}} I_{\left\{\tau_{p}>s\right\}} \mathrm{d} s\right] \\
& +E_{t}^{Q}\left[M\left(\tau_{d}\right) R\left(\tau_{d}\right) \mathrm{e}^{-\int_{t}^{\tau_{p}} r(u) \mathrm{d} u} I_{\left\{\tau_{d}<T\right\}}\right]+E_{t}^{Q}\left[\int_{t}^{T} L \mathrm{e}^{-\int_{t}^{s} r(u) \mathrm{d} u} I_{\left\{\tau_{d}<T\right\}} I_{\left\{\tau_{d}>S\right\}} \mathrm{d} s\right] \\
& +E_{t}^{Q}\left[\int_{t}^{T} L \mathrm{e}^{-\int_{t}^{S} r(u) \mathrm{d} u} I_{\left\{\tau_{p}>T, \tau_{d}>T\right\}} I_{\{\tau>s\}} \mathrm{d} s\right] .
\end{aligned}
$$


Simplifying it, we have

$$
\begin{aligned}
V(t)= & E_{t}^{Q}\left[M\left(\tau_{p}\right) \mathrm{e}^{-\int_{t}^{\tau} p r(u) d u} I_{\left\{\tau_{p}<T\right\}}\right]+E_{t}^{Q}\left[M\left(\tau_{d}\right) R\left(\tau_{d}\right) \mathrm{e}^{-\int_{t}^{\tau_{d}} r(u) \mathrm{d} u} I_{\left\{\tau_{p}<T\right\}}\right] \\
& +E_{t}^{Q}\left[\int_{t}^{T} L \mathrm{e}^{-\int_{t}^{s} r(u) \mathrm{d} u} I_{\{\tau>>s\}} \mathrm{d} s\right],
\end{aligned}
$$

where $E_{t}^{Q}[\cdot]=E^{Q}\left[\cdot \mid \mathcal{F}_{t}\right]$. Now, we give a lemma about the distributions of $\tau_{d}^{i}$ and $\tau_{p}^{i}$.

Lemma 1. For all $T \in\left[t, T^{*}\right]$, the conditional probabilities of the default and the prepayment of firm $i$ are respectively

$$
\begin{aligned}
& P\left(\tau_{d}^{i}>T \mid \mathcal{G}_{t}^{i}\right)=I_{\left\{\tau_{d}^{i}>t\right\}} \exp \left(-\int_{t}^{T} \lambda^{i}(u) \mathrm{d} u\right) \\
& P\left(\tau_{p}^{i}>T \mid \mathcal{G}_{t}^{i}\right)=I_{\left\{\tau_{p}^{i}>t\right\}} E_{t}^{Q}\left[\mathrm{e}^{-\int_{t}^{T} \xi^{i}(u) \mathrm{d} u}\right] .
\end{aligned}
$$

Proof. The process in details can be found in Appendix.

In order to calculate the price of the loan, we give another form of the pricing formula (8).

Theorem 1. The pricing formula of the loan $V^{i}(t)$ has the following form

$$
\begin{aligned}
V^{i}(t)= & E_{t}^{Q}\left[\int_{t}^{T} M(s) \xi^{i}(s) \mathrm{e}^{-\int_{t}^{s}\left(r(u)+\xi^{i}(u)+\lambda^{i}(u)\right) \mathrm{d} u} \mathrm{~d} s\right] \\
& +E_{t}^{Q}\left[\int_{t}^{T} R(s) M(s) \lambda^{i}(s) \mathrm{e}^{-\int_{t}^{s}\left(r(u)+\xi^{i}(u)+\lambda^{i}(u)\right) \mathrm{d} u} \mathrm{~d} s\right] \\
& +L E_{t}^{Q}\left[\int_{t}^{T} \mathrm{e}^{-\int_{t}^{s}\left(r(u)+\xi^{i}(u)+\lambda^{i}(u)\right) \mathrm{d} u} \mathrm{~d} s\right] .
\end{aligned}
$$

Proof. The process in details can be found in Appendix.

In the following, we give the primary-secondary model with the attenuation effect which the intensities of the default and the prepayment of firm $A$ and firm $B$ satisfy. We apply the contagious model into the loan and loan CDS based on the above loan.

Suppose that the default intensity and the intensity of repaying the loan in advance of the primary firm $A$ satisfy the following equations:

$$
\lambda^{A}(t)=a_{0}^{A}+a_{1}^{A} r(t), \xi^{A}(t)=b_{0}^{A}
$$

the default intensity and the intensity of repaying the loan in advance of the secondary firm $B$ satisfy the following equations:

$$
\lambda^{B}(t)=a_{0}^{B}+a_{1}^{B} r(t)+I_{\left\{\tau_{d}^{A}<t\right\}} \frac{a_{2}^{B}}{a_{3}^{B}\left(t-\tau_{d}^{A}\right)+1}, \xi^{B}(t)=b_{0}^{B},
$$

where $a_{0}^{A}, a_{1}^{A}, a_{0}^{B}, a_{1}^{B}, a_{3}^{B}, b_{0}^{A}, b_{0}^{B}>0, a_{2}^{B}$ are real and $a_{0}^{A}+a_{1}^{A}>0, \quad a_{0}^{B}+a_{1}^{B}+a_{2}^{B}>0$.

Now, we assume that the interest rate satisfies Vasicek model,

$$
\mathrm{d} r(t)=a(b-r(t)) \mathrm{d} t+\sigma_{r} \mathrm{~d} Z_{r}(t),
$$

where $Z_{r}(t)$ is the standard Brownian motion which describes the market risk, $\sigma_{r}$ is the standard deviation which represents the stochastic volatility, parameter $b$ is the long-term average of interest rate, $a$ represents the speed of recovery that $r_{t}$ returns to $b$ from the deviation value of the long-term average. The interest rate has the following explicit solution:

$$
r(u)=f(t, u)+\frac{\sigma_{r}^{2}\left(\mathrm{e}^{-a(u-t)}-1\right)^{2}}{2 a^{2}}+\int_{t}^{u} \sigma_{r} \mathrm{e}^{-a(u-v)} \mathrm{d} Z_{r}(v) .
$$

Let $\rho(v, u)=\sigma_{r} \mathrm{e}^{-a(u-v)}, b(t, u)=\int_{t}^{u} \rho(t, v) \mathrm{d} v=\sigma_{r} \frac{\left(1-\mathrm{e}^{-a(u-t)}\right)}{a}$. From [7], we have 


$$
\begin{aligned}
& \int_{t}^{s} r(u) \mathrm{d} u=\int_{t}^{s} f(t, u) \mathrm{d} u+\int_{t}^{s} \frac{b(t, u)^{2}}{2} \mathrm{~d} u+\int_{t}^{s} b(v, s) \mathrm{d} Z_{r}(v), \\
& E_{t}^{Q}\left[\int_{t}^{s} r(u) \mathrm{d} u\right] \\
&=\int_{t}^{s} f(t, u) \mathrm{d} u+\int_{t}^{s} \frac{b(t, u)^{2}}{2} \mathrm{~d} u \\
&=f(t, s)(s-t)+\frac{\sigma_{r}^{2}}{2 a^{2}}\left((s-t)-\frac{2}{a}\left(1-\mathrm{e}^{-a(s-t)}\right)+\frac{1}{2 a}\left(1-\mathrm{e}^{-2 a(s-t)}\right)\right), \\
& \quad \operatorname{Var}_{t}\left(\int_{t}^{s} b(v, s) \mathrm{d} Z_{r}(v)\right) \\
&=E_{t}^{Q}\left[\int_{t}^{s} b(v, s) \mathrm{d} Z_{r}(v) \times \int_{t}^{s} b(v, s) \mathrm{d} Z_{r}(v)\right]=\int_{t}^{s} b(v, s)^{2} \mathrm{~d} v \\
&=\frac{\sigma_{r}^{2}}{a^{2}}\left((s-t)-\frac{2}{a}\left(1-\mathrm{e}^{-a(s-t)}\right)+\frac{1}{2 a}\left(1-\mathrm{e}^{-2 a(s-t)}\right)\right) .
\end{aligned}
$$

\subsection{The Price of the Loan Issued by the Primary Firm $A$}

In fact, we need to substitute (11) into the pricing formula in Theorem 1.

Firstly, we calculate $L E_{t}^{Q}\left[\int_{t}^{T} \mathrm{e}^{-\int_{t}^{s}\left(r(u)+\xi^{A}(u)+\lambda^{A}(u)\right) \mathrm{d} u} \mathrm{~d} s\right]$.

$$
\begin{aligned}
& L E_{t}^{Q}\left[\int_{t}^{T} \mathrm{e}^{-\int_{t}^{S}\left(r(u)+\xi^{A}(u)+\lambda^{A}(u)\right) \mathrm{d} u} \mathrm{~d} s\right] \\
& =L \int_{t}^{T} E_{t}^{Q}\left[\mathrm{e}^{-\int_{t}^{S}\left(r(u)+\xi^{A}(u)+\lambda^{A}(u)\right) \mathrm{d} u}\right] \\
& =L \int_{t}^{T} E_{t}^{Q}\left[\mathrm{e}^{-\int_{t}^{S}\left(r(u)+a_{0}^{A}+b_{0}^{A}+a_{1}^{A} r(u)\right) \mathrm{d} u}\right] \\
& =L \int_{t}^{T} \mathrm{e}^{-(s-t)\left(a_{0}^{A}+b_{0}^{A}\right)} E_{t}^{Q}\left[\mathrm{e}^{-\left(1+a_{1}^{A}\right) \int_{t}^{S} r(u) \mathrm{d} u}\right] \mathrm{d} s .
\end{aligned}
$$

For any normal random variables $X$ and $Y$,

$$
E\left(\mathrm{e}^{A X+B Y}\right)=\exp \left[A \mu_{X}+B \mu_{Y}+\frac{1}{2}\left(A^{2} \sigma_{X}^{2}+2 \sigma_{X Y} A B+B^{2} \sigma_{Y}^{2}\right)\right] .
$$

So we obtain

$$
\begin{aligned}
& E_{t}^{Q}\left[\mathrm{e}^{-\left(1+a_{1}^{A}\right) \int_{t}^{s} r(u) \mathrm{d} u}\right]=\exp \left[-\left(1+a_{1}^{A}\right) E_{t}^{Q}\left(\int_{t}^{s} r(u) \mathrm{d} u\right)+\frac{1}{2} \operatorname{Var}_{t}\left(\int_{t}^{s} r(u) \mathrm{d} u\right)\right] \\
& =\exp \left[-\left(1+a_{1}^{A}\right)(f(t, s)(s-t))-\frac{a_{1}^{A} \sigma_{r}^{2}}{2 a^{2}}\left((s-t)-\frac{2}{a}\left(1-\mathrm{e}^{-a(s-t)}\right)+\frac{1}{2 a}\left(1-\mathrm{e}^{-2 a(s-t)}\right)\right)\right] .
\end{aligned}
$$

Secondly, we calculate $E_{t}^{Q}\left[\int_{t}^{T} M(s) \xi^{A}(s) \mathrm{e}^{-\int_{t}^{s}\left(r(u)+\xi^{A}(u)+\lambda^{A}(u)\right) \mathrm{d} u} \mathrm{~d} s\right]$.

$$
\begin{aligned}
& E_{t}^{Q}\left[\int_{t}^{T} M(s) \xi^{A}(s) \mathrm{e}^{-\int_{t}^{S}\left(r(u)+\xi^{A}(u)+\lambda^{A}(u)\right) \mathrm{d} u} \mathrm{~d} s\right] \\
& =\int_{t}^{T} M(s) b_{0}^{A} E_{t}^{Q}\left[\mathrm{e}^{-\int_{t}^{S}\left(r(u)+\xi^{A}(u)+\lambda^{A}(u)\right) \mathrm{d} u} \mathrm{~d} s\right],
\end{aligned}
$$

then, we substitute (18) into (21) and get the value of $E_{t}^{Q}\left[\int_{t}^{T} M(s) \xi^{A}(s) \mathrm{e}^{-\int_{t}^{s}\left(r(u)+\xi^{A}(u)+\lambda^{A}(u)\right) d u} \mathrm{~d} s\right]$. 
At last, we calculate $E_{t}^{Q}\left[\int_{t}^{T} R(s) M(s) \lambda^{A}(s) \mathrm{e}^{-\int_{t}^{s}\left(r(u)+\xi^{A}(u)+\lambda^{A}(u)\right) d u} \mathrm{~d} s\right]$.

$$
\begin{aligned}
& E_{t}^{Q}\left[\int_{t}^{T} R(s) M(s) \lambda^{A}(s) \mathrm{e}^{-\int_{t}^{s}\left(r(u)+\xi^{A}(u)+\lambda^{A}(u)\right) \mathrm{d} u} \mathrm{~d} s\right] \\
& =E_{t}^{Q}\left[\int_{t}^{T} R(s) M(s)\left(a_{0}^{A}+a_{1}^{A} r(s)\right) \mathrm{e}^{-\int_{t}^{s}\left(r(u)+\xi^{A}(u)+\lambda^{A}(u)\right) \mathrm{d} u} \mathrm{~d} s\right] \\
& =\int_{t}^{T} R(s) M(s) a_{0}^{A} E_{t}^{Q}\left[\mathrm{e}^{-\int_{t}^{s}\left(r(u)+\xi^{A}(u)+\lambda^{A}(u)\right) \mathrm{d} u}\right] \mathrm{d} s \\
& +\int_{t}^{T} R(s) M(s) a_{1}^{A} r(s) E_{t}^{Q}\left[\mathrm{e}^{-\int_{t}^{s}\left(r(u)+\xi^{A}(u)+\lambda^{A}(u)\right) \mathrm{d} u}\right] \mathrm{d} s \\
& =\int_{t}^{T} R(s) M(s) a_{0}^{A} E_{t}^{Q}\left[\mathrm{e}^{-\int_{t}^{s}\left(r(u)+\xi^{A}(u)+\lambda^{A}(u)\right) \mathrm{d} u}\right] \mathrm{d} s \\
& +\int_{t}^{T} R(s) M(s) a_{1}^{A} \mathrm{e}^{-(s-t)\left(a_{0}^{A}+b_{0}^{A}\right)} E_{t}^{Q}\left[r(s) \mathrm{e}^{-\left(1+a_{1}^{A}\right) f_{r}^{S} r(u) \mathrm{d} u}\right] \mathrm{d} s .
\end{aligned}
$$

For any normal random variables $X$ and $Y$,

$$
\begin{aligned}
& \frac{\partial E\left[\mathrm{e}^{A X+B Y}\right]}{\partial B}=E\left[B \mathrm{e}^{A X+B Y}\right] \\
& =\exp \left[A \mu_{X}+B \mu_{Y}+\frac{1}{2}\left(A^{2} \sigma_{X}^{2}+2 A B \sigma_{X Y}+B^{2} \sigma_{Y}^{2}\right)\right]\left(\mu_{Y}+B \sigma_{Y}^{2}+A \sigma_{X Y}\right) .
\end{aligned}
$$

Let $X=\int_{t}^{s} r(u) \mathrm{d} u, Y=r(s)$. We have

$$
\begin{aligned}
E_{t}^{Q}\left[r(s) \mathrm{e}^{-\left(1+a_{1}^{A}\right) \int_{t}^{s} r(u) \mathrm{d} u}\right] & =\left.\frac{\partial E_{t}^{Q}\left[\mathrm{e}^{-\left(1+a_{1}^{A}\right) \int_{t}^{s} r(u) \mathrm{d} u+B r(s)}\right]}{\partial B}\right|_{B=0} \\
& =\exp \left(A \mu_{X}+\frac{1}{2} A^{2} \sigma_{X}^{2}\right)\left(\mu_{Y}+A \sigma_{X Y}\right)
\end{aligned}
$$

and

$$
\begin{aligned}
\sigma_{X Y} & =\operatorname{Cov}_{t}\left(r(s), \int_{t}^{s} r(u) \mathrm{d} u\right) \\
& =E_{t}^{Q}\left[r(s) \int_{t}^{s} r(u) \mathrm{d} u\right]-E_{t}^{Q}[r(s)] E_{t}^{Q}\left[\int_{t}^{s} r(u) \mathrm{d} u\right] \\
& =E_{t}^{Q}\left[\int_{t}^{s} \rho(v, s) \mathrm{d} Z_{r}(v) \times \int_{t}^{s} b(v, s) \mathrm{d} Z_{r}(v)\right] \\
& =\int_{t}^{s} \rho(v, s) b(v, s) \mathrm{d} v \\
& =\frac{\sigma_{r}^{2}}{2 a^{2}}\left(1-\mathrm{e}^{-a(s-t)}\right)^{2} .
\end{aligned}
$$

Substituting (18), (24) and (25) into (22), we deduce the price of the loan issued by the primary firm $A$.

\subsection{The Price of the Loan Issued by the Secondary Firm $B$}

The pricing process is similar to the pricing of the primary firm $A$. We need three steps.

Firstly, we calculate $L E_{t}^{Q}\left[\int_{t}^{T} \mathrm{e}^{-\int_{t}^{s}\left(r(u)+\xi^{B}(u)+\lambda^{B}(u)\right) \mathrm{d} u} \mathrm{~d} s\right]$. 


$$
\begin{aligned}
& L E_{t}^{Q}\left[\int_{t}^{T} \mathrm{e}^{-\int_{t}^{S}\left(r(u)+\xi^{B}(u)+\lambda^{B}(u)\right) \mathrm{d} u} \mathrm{~d} s\right]=L \int_{t}^{T} E_{t}^{Q}\left[\mathrm{e}^{-\int_{t}^{s}\left(r(u)+\xi^{B}(u)+\lambda^{B}(u)\right) \mathrm{d} u}\right] \mathrm{d} s \\
& =L \int_{t}^{T} E_{t}^{Q}\left[\mathrm{e}^{-\int_{t}^{s}\left(r(u)+b_{0}^{B}+a_{0}^{B}+a_{1}^{B} r(u)+I_{\left\{\tau_{d}^{A}<u\right\}} \frac{a_{2}^{B}}{a_{3}^{B}\left(u-\tau_{d}^{A}\right)+1}\right)} d u d \mathrm{~d} s\right. \\
& =L \int_{t}^{T} E_{t}^{Q}\left[\mathrm{e}^{-\int_{t}^{s}\left(r(u)+b_{0}^{B}+a_{0}^{B}+a_{1}^{B} r(u)\right) \mathrm{d} u} E^{Q}\left(\mathrm{e}^{-\int_{t}^{s}\left(I_{\left\{\tau_{d}^{A}<u\right\}} \frac{a_{2}^{B}}{a_{3}^{B}\left(u-\tau_{d}^{A}\right)+1}\right) \mathrm{d} u} \mid \mathcal{F}_{t} \vee \mathcal{F}_{T^{*}}^{r}\right)\right] \mathrm{d} s .
\end{aligned}
$$

Let $-a_{2}^{B}=a_{3}^{B}=C^{A}>0$, then

$$
\begin{aligned}
& E^{Q}\left(\mathrm{e}^{-\int_{t}^{s}\left(I_{\left\{\tau_{d}^{A}<u\right\}} \frac{-C^{A}}{C^{A}\left(u-\tau_{d}^{A}\right)+1}\right) \mathrm{d} u} \mid \mathcal{F}_{t} \vee \mathcal{F}_{T^{*}}^{r}\right) \\
& =E^{Q}\left(\exp \left(-\left(\int_{t}^{\tau_{d}^{A}}+\int_{\tau_{d}^{A}}^{s} I_{\left\{\tau_{d}^{A}<u\right\}} \frac{-C^{A}}{C^{A}\left(u-\tau_{d}^{A}\right)+1}\right) \mathrm{d} u\right) \mid \mathcal{F}_{t} \vee \mathcal{F}_{T^{*}}^{r}\right) \\
& =E^{Q}\left(\exp \left(I_{\left\{t<\tau_{d}^{A}<s\right\}} \ln \left[C^{A}\left(s-\tau_{d}^{A}\right)+1\right]\right) \mid \mathcal{F}_{t} \vee \mathcal{F}_{T^{*}}^{r}\right) \\
& =\int_{t}^{s}+\int_{s}^{\infty} \exp \left(I_{\{t<u<s\}} \ln \left[C^{A}(s-u)+1\right]\right) \mathrm{d}\left(1-P\left(\tau_{d}^{A}>u \mid \mathcal{F}_{t} \vee \mathcal{F}_{T^{*}}^{r}\right)\right) \\
& =\int_{t}^{s}\left[C^{A}(s-u)+1\right] d\left(1-P\left(\tau_{d}^{A}>u \mid \mathcal{F}_{t} \vee \mathcal{F}_{T^{*}}^{r}\right)\right)-\int_{s}^{\infty} \mathrm{d}\left(P\left(\tau_{d}^{A}>u \mid \mathcal{F}_{t} \vee \mathcal{F}_{T^{*}}^{r}\right)\right) .
\end{aligned}
$$

In the above equation, applying the integration by parts,

$$
\begin{aligned}
\int_{t}^{s} & {\left[C^{A}(s-u)+1\right] \mathrm{d}\left(1-P\left(\tau_{d}^{A}>u \mid \mathcal{F}_{t} \vee \mathcal{F}_{T^{*}}^{r}\right)\right)-\int_{s}^{\infty} \mathrm{d}\left(P\left(\tau_{d}^{A}>u \mid \mathcal{F}_{t} \vee \mathcal{F}_{T^{*}}^{r}\right)\right) } \\
= & {\left.\left[C^{A}(s-u)+1\right]\left(1-P\left(\tau_{d}^{A}>u \mid \mathcal{F}_{t} \vee \mathcal{F}_{T^{*}}^{r}\right)\right)\right\}_{t}^{s} } \\
& +C^{A} \int_{t}^{s}\left(1-P\left(\tau_{d}^{A}>u \mid \mathcal{F}_{t} \vee \mathcal{F}_{T^{*}}^{r}\right)\right) \mathrm{d} u+P\left(\tau_{d}^{A}>s \mid \mathcal{F}_{t} \vee \mathcal{F}_{T^{*}}^{r}\right) \\
= & \left(1-P\left(\tau_{d}^{A}>s \mid \mathcal{F}_{t} \vee \mathcal{F}_{T^{*}}^{r}\right)\right)+C^{A}(s-t) \\
& -C^{A} \int_{t}^{s} P\left(\tau_{d}^{A}>u \mid \mathcal{F}_{t} \vee \mathcal{F}_{T^{*}}^{r}\right) \mathrm{d} u+P\left(\tau_{d}^{A}>s \mid \mathcal{F}_{t} \vee \mathcal{F}_{T^{*}}^{r}\right) \\
= & C^{A}(s-t)+1-C^{A} \int_{t}^{s} P\left(\tau_{d}^{A}>u \mid \mathcal{F}_{t} \vee \mathcal{F}_{T^{*}}^{r}\right) \mathrm{d} u .
\end{aligned}
$$

We assume that no defaults occur up to time $t$ for firm $A$. Then

$$
P\left(\tau_{d}^{A}>u \mid \mathcal{F}_{t} \vee \mathcal{F}_{T^{*}}^{r}\right)=\exp \left(-\int_{t}^{u} \lambda^{A}(v) \mathrm{d} v\right)=\exp \left(-a_{0}^{A}(u-t)-a_{1}^{A} \int_{t}^{u} r(v) \mathrm{d} v\right) .
$$

Substituting (29) into (28), we obtain

$$
\begin{aligned}
& C^{A}(s-t)+1-C^{A} \int_{t}^{s} P\left(\tau_{d}^{A}>u \mid \mathcal{F}_{t} \vee \mathcal{F}_{T^{*}}^{r}\right) \mathrm{d} u \\
& =C^{A}(s-t)+1-C^{A} \int_{t}^{s} \exp \left(-a_{0}^{A}(u-t)-a_{1}^{A} \int_{t}^{u} r(v) \mathrm{d} v\right) \mathrm{d} u .
\end{aligned}
$$

By (26), 


$$
\begin{aligned}
& L \int_{t}^{T} E_{t}^{Q}\left[\mathrm{e}^{-\int_{t}^{s}\left(r(u)+b_{0}^{B}+a_{0}^{B}+a_{1}^{B} r(u)\right) \mathrm{d} u} E\left(\mathrm{e}^{-\int_{t}^{s}\left(I_{\left\{t_{d}^{A} u u\right\}} \frac{a_{2}^{B}}{a_{3}^{B}\left(u-\tau_{d}^{A}\right)+1}\right) \mathrm{d} u} \mid \mathcal{F}_{t} \vee \mathcal{F}_{T^{*}}^{r}\right)\right] \mathrm{d} s \\
= & L \int_{t}^{T} E_{t}^{Q}\left[\mathrm{e}^{-\int_{t}^{s}\left(r(u)+b_{0}^{B}+a_{0}^{B}+a_{1}^{B} r(u)\right) \mathrm{d} u}\left(C^{A}(s-t)+1-C^{A} \int_{t}^{s} \exp \left(-a_{0}^{A}(u-t)-a_{1}^{A} \int_{t}^{u} r(v) \mathrm{d} v\right) \mathrm{d} u\right)\right] \mathrm{d} s \\
= & L \int_{t}^{T} E_{t}^{Q}\left[\mathrm{e}^{\left(b_{0}^{B}+a_{0}^{B}\right)(s-t)} \mathrm{e}^{-\int_{t}^{s}\left(1+a_{1}^{B}\right) r(u) \mathrm{d} u}\left(C^{A}(s-t)+1-C^{A} \int_{t}^{s} \exp \left(-a_{0}^{A}(u-t)-a_{1}^{A} \int_{t}^{u} r(v) \mathrm{d} v\right) \mathrm{d} u\right)\right] \mathrm{d} s \\
= & L \int_{t}^{T} E_{t}^{Q}\left[\mathrm{e}^{\left(b_{0}^{B}+a_{0}^{B}\right)(s-t)} \mathrm{e}^{-\int_{t}^{s}\left(1+a_{1}^{B}\right) r(u) \mathrm{d} u}\left(C^{A}(s-t)+1\right)\right] \mathrm{d} s \\
& -L \int_{t}^{T} C^{A} E_{t}^{Q}\left[\mathrm{e}^{\left(b_{0}^{B}+a_{0}^{B}\right)(s-t)} \mathrm{e}^{-\int_{t}^{s}\left(1+a_{1}^{B}\right) r(u) \mathrm{d} u}\left(\int_{t}^{s} \exp \left(-a_{0}^{A}(u-t)-a_{1}^{A} \int_{t}^{u} r(v) \mathrm{d} v\right) \mathrm{d} u\right)\right] \mathrm{d} s \\
= & L \int_{t}^{T}\left(C^{A}(s-t)+1\right) \mathrm{e}^{\left(b_{0}^{B}+a_{0}^{B}\right)(s-t)} E_{t}^{Q}\left[\mathrm{e}^{-\int_{t}^{s}\left(1+a_{1}^{B}\right) r(u) \mathrm{d} u}\right] \mathrm{d} s-L \int_{t}^{T} C^{A} \mathrm{e}^{\left(b_{0}^{B}+a_{0}^{B}\right)(s-t)} E_{t}^{Q}\left[\mathrm{e}^{-\int_{t}^{s}\left(1+a_{1}^{B}\right) r(u)}\left(\int_{t}^{s} \mathrm{e}^{-a_{0}^{A}(u-t)-a_{1}^{A} \int_{t}^{u} r(v) \mathrm{d} v} \mathrm{~d} u\right)\right] \mathrm{d} s \\
= & L \int_{t}^{T}\left(C^{A}(s-t)+1\right) \mathrm{e}^{\left(b_{0}^{B}+a_{0}^{B}\right)(s-t)} E_{t}^{Q}\left[\mathrm{e}^{-\left(1+a_{1}^{B}\right) \int_{t}^{s} r(u) \mathrm{d} u}\right] \mathrm{d} s-L \int_{t}^{T} C^{A} \mathrm{e}^{\left(b_{0}^{B}+a_{0}^{B}\right)(s-t)} \int_{t}^{s} \mathrm{e}^{-a_{0}^{A}(u-t)} E_{t}^{Q}\left[\mathrm{e}^{\left.-\left(1+a_{1}^{B}\right) \int_{t}^{s} r(u) \mathrm{d} u \mathrm{e}^{-a_{1}^{A} \int_{t}^{u} r(v) \mathrm{d} v}\right] \mathrm{d} u \mathrm{~d} s .}\right.
\end{aligned}
$$

Then, applying the conclusion that $E\left(\mathrm{e}^{A X+B Y}\right)=\exp \left[A \mu_{X}+B \mu_{Y}+\frac{1}{2}\left(A^{2} \sigma_{X}^{2}+2 \sigma_{X Y} A B+B^{2} \sigma_{Y}^{2}\right)\right]$ for any normal random variables $X$ and $Y$, we have (31).

Secondly, we calculate $E_{t}^{Q}\left[\int_{t}^{T} M(s) \xi^{B}(s) \mathrm{e}^{-\int_{t}^{s}\left(r(u)+\xi^{B}(u)+\lambda^{B}(u)\right) \mathrm{d} u} \mathrm{~d} s\right]$.

$$
E_{t}^{Q}\left[\int_{t}^{T} M(s) \xi^{B}(s) \mathrm{e}^{-\int_{t}^{s}\left(r(u)+\xi^{B}(u)+\lambda^{B}(u)\right) \mathrm{d} u} \mathrm{~d} s\right]=\int_{t}^{T} M(s) b_{0}^{B} E_{t}^{Q}\left[\mathrm{e}^{-\int_{t}^{s}\left(r(u)+\xi^{B}(u)+\lambda^{B}(u)\right) \mathrm{d} u}\right] \mathrm{d} s .
$$

Because $E_{t}^{Q}\left[\mathrm{e}^{-\int_{t}^{s}\left(r(u)+\xi^{B}(u)+\lambda^{B}(u)\right) \mathrm{d} u}\right]$ is calculated in the first step, (32) can be easily obtained.

At last, we calculate $E_{t}^{Q}\left[\int_{t}^{T} R(s) M(s) \lambda^{B}(s) \mathrm{e}^{-\int_{t}^{S}\left(r(u)+\xi^{B}(u)+\lambda^{B}(u)\right) \mathrm{d} u} \mathrm{~d} s\right]$.

$$
\begin{aligned}
& \int_{t}^{T} R(s) M(s) \lambda^{B}(s) \mathrm{e}^{-\int_{t}^{s}\left(r(u)+\xi^{B}(u)+\lambda^{B}(u)\right) \mathrm{d} u} \mathrm{~d} s \\
& =\int_{t}^{T} R(s) M(s)\left(r(s)+\xi^{B}(s)+\lambda^{B}(s)\right) \mathrm{e}^{-\int_{t}^{s}\left(r(u)+\xi^{B}(u)+\lambda^{B}(u)\right) \mathrm{d} u} \mathrm{~d} s \\
& -\int_{t}^{T} R(s) M(s)\left(r(s)+\xi^{B}(s)\right) \mathrm{e}^{-\int_{t}^{s}\left(r(u)+\xi^{B}(u)+\lambda^{B}(u)\right) \mathrm{d} u} \mathrm{~d} s \\
& =\int_{t}^{T} R(s) M(s) \mathrm{d}\left(-\mathrm{e}^{-\int_{t}^{S}\left(r(u)+\xi^{B}(u)+\lambda^{B}(u)\right) \mathrm{d} u}\right)-\int_{t}^{T} R(s) M(s)\left(r(s)+\xi^{B}(s)\right) \mathrm{e}^{-\int_{t}^{s}\left(r(u)+\xi^{B}(u)+\lambda^{B}(u)\right) \mathrm{d} u} \mathrm{~d} s \\
& =-\left.R(s) M(s) \mathrm{e}^{-\int_{t}^{s}\left(r(u)+\xi^{B}(u)+\lambda^{B}(u)\right) d u}\right|_{t} ^{T}+\int_{t}^{T} \mathrm{e}^{-\int_{t}^{s}\left(r(u)+\xi^{B}(u)+\lambda^{B}(u)\right) d u} \mathrm{~d}(R(s) M(s)) \\
& -\int_{t}^{T} R(s) M(s)\left(r(s)+\xi^{B}(s)\right) \mathrm{e}^{-\int_{t}^{s}\left(r(u)+\xi^{B}(u)+\lambda^{B}(u)\right) \mathrm{d} u} \mathrm{~d} s \\
& =-\left(\mathrm{e}^{-\int_{t}^{T}\left(r(u)+\xi^{B}(u)+\lambda^{B}(u)\right) \mathrm{d} u} R(T) M(T)-R(t) M(t)-\int_{t}^{T} \mathrm{e}^{-\int_{t}^{T}\left(r(u)+\xi^{B}(u)+\lambda^{B}(u)\right) \mathrm{d} u}(R(s) M(s))^{\prime} \mathrm{d} s\right) \\
& -\int_{t}^{T} R(s) M(s)\left(r(s)+\xi^{B}(s)\right) \mathrm{e}^{-\int_{t}^{s}\left(r(u)+\xi^{B}(u)+\lambda^{B}(u)\right) d u} \mathrm{~d} s .
\end{aligned}
$$


So

$$
\begin{aligned}
E_{t}^{Q} & {\left[\int_{t}^{T} R(s) M(s) \lambda^{B}(s) \mathrm{e}^{-\int_{t}^{s}\left(r(u)+\xi^{B}(u)+\lambda^{B}(u)\right) \mathrm{d} u} \mathrm{~d} s\right] } \\
= & R(t) M(t)+\int_{t}^{T}(R(s) M(s))^{\prime} E_{t}^{Q}\left[\mathrm{e}^{-\int_{t}^{T}\left(r(u)+\xi^{B}(u)+\lambda^{B}(u)\right) \mathrm{d} u}\right] \mathrm{d} s \\
& -\int_{t}^{T} R(s) M(s) E_{t}^{Q}\left[r(s) \mathrm{e}^{-\int_{t}^{s}\left(r(u)+\xi^{B}(u)+\lambda^{B}(u)\right) \mathrm{d} u}\right] \mathrm{d} s \\
& -\int_{t}^{T} R(s) M(s) E_{t}^{Q}\left[\xi^{B}(s) \mathrm{e}^{-\int_{t}^{S}\left(r(u)+\xi^{B}(u)+\lambda^{B}(u)\right) \mathrm{d} u}\right] \mathrm{d} s .
\end{aligned}
$$

From above equation, we only need calculate $\int_{t}^{T} R(s) M(s) E_{t}^{Q}\left[r(s) \mathrm{e}^{-\int_{t}^{s}\left(r(u)+\xi^{B}(u)+\lambda^{B}(u)\right) d u}\right] \mathrm{d} s \quad$ in (33).

$$
\begin{aligned}
& E_{t}^{Q}\left[r(s) \mathrm{e}^{-\int_{t}^{s}\left(r(u)+\xi^{B}(u)+\lambda^{B}(u)\right) \mathrm{d} u}\right] \\
& =E_{t}^{Q}\left[r(s) \mathrm{e}^{-\int_{t}^{s}\left(r(u)+b_{0}^{B}+a_{0}^{B}+a_{1}^{B} r(u)\right) \mathrm{d} u} E\left(\mathrm{e}^{-\int_{t}^{S}\left(\int_{\left\{\tau_{d}^{A}<u\right\}} \frac{a_{2}^{B}}{a_{3}^{B}\left(u-\tau_{d}^{A}\right)+1}\right) \mathrm{d} u} \mid \mathcal{F}_{t} \vee \mathcal{F}_{T^{*}}^{r}\right)\right] \\
& =\left(C^{A}(s-t)+1\right) E_{t}^{Q}\left[r(s) \mathrm{e}^{-\left(1+a_{1}^{B}\right) \int_{t}^{s} r(u) \mathrm{d} u}\right]-C^{A} \int_{t}^{s} \mathrm{e}^{-a_{0}^{A}(u-t)} E_{t}^{Q}\left[r(s) \mathrm{e}^{-\left(1+a_{1}^{B}\right) \int_{t}^{S} r(u) \mathrm{d} u} \mathrm{e}^{-a_{1}^{A} \int_{t}^{u} r(v) \mathrm{d} v}\right] \mathrm{d} u .
\end{aligned}
$$

The first part in (35) is similarly obtained by the same method and process to the former, so we omit it. Now, we calculate the second part. Let $X=-\left(1+a_{1}^{B}\right) \int_{t}^{s} r(u) \mathrm{d} u-a_{1}^{A} \int_{t}^{u} r(v) \mathrm{d} v, Y=r(s)$, then

$$
\begin{aligned}
& E_{t}^{Q}\left[r(s) \mathrm{e}^{-\left(1+a_{1}^{B}\right) \int_{t}^{s} r(u) \mathrm{d} u} \mathrm{e}^{-a_{1}^{A} \int_{t}^{u} r(v) \mathrm{d} v}\right] \\
& =\left.\frac{\partial E_{t}^{Q}\left[\mathrm{e}^{-\left(1+a_{1}^{B}\right) \int_{t}^{s} r(u) \mathrm{d} u+B r(s)-a_{1}^{A} \int_{t}^{u} r(v) \mathrm{d} v}\right]}{\partial B}\right|_{B=0}=\exp \left(A \mu_{X}+\frac{1}{2} A^{2} \sigma_{X}^{2}\right)\left(\mu_{Y}+A \sigma_{X Y}\right),
\end{aligned}
$$

where $\mu_{X}$ and $\mu_{Y}$ are easily obtained by the conclusions about the interest rate $r(t)$. For $\sigma_{X Y}$, we have

$$
\begin{aligned}
\sigma_{X Y} & =\operatorname{Cov}_{t}\left(r(s),-\left(1+a_{1}^{B}\right) \int_{t}^{s} r(u) \mathrm{d} u-a_{1}^{A} \int_{t}^{u} r(v) \mathrm{d} v\right) \\
& =-\left(1+a_{1}^{B}\right) \operatorname{Cov}_{t}\left(r(s), \int_{t}^{s} r(u) \mathrm{d} u\right)-a_{1}^{A} \operatorname{Cov}_{t}\left(r(s), \int_{t}^{u} r(v) \mathrm{d} v\right) \\
& =-\left(1+a_{1}^{B}\right) E_{t}^{Q}\left[\int_{t}^{s} \rho(v, s) \mathrm{d} Z_{r}(v) \times \int_{t}^{s} b(v, s) \mathrm{d} Z_{r}(v)\right]-a_{1}^{A} E_{t}^{Q}\left[\int_{t}^{s} \rho(v, s) \mathrm{d} Z_{r}(v) \times \int_{t}^{u} b(v, u) \mathrm{d} Z_{r}(v)\right] \\
& =-\left(1+a_{1}^{B}\right) \int_{t}^{s} \rho(v, s) b(v, s) \mathrm{d} v-a_{1}^{A} \int_{t}^{u} \rho(v, s) b(v, u) \mathrm{d} v .
\end{aligned}
$$

For $\sigma_{X}^{2}, u<s$, we have

$$
\begin{aligned}
& \sigma_{X}^{2}=\operatorname{Var}_{t}\left(-\left(1+a_{1}^{B}\right) \int_{t}^{s} r(u) \mathrm{d} u-a_{1}^{A} \int_{t}^{u} r(v) \mathrm{d} v\right) \\
& =\left(1+a_{1}^{B}\right)^{2} \operatorname{Var}_{t}\left(\int_{t}^{s} r(u) \mathrm{d} u\right)+\left(a_{1}^{A}\right)^{2} \operatorname{Var}_{t}\left(\int_{t}^{u} r(v) \mathrm{d} v\right)+2 a_{1}^{A}\left(1+a_{1}^{B}\right) \operatorname{Cov}_{t}\left(\int_{t}^{s} r(u) \mathrm{d} u, \int_{t}^{u} r(v) \mathrm{d} v\right) \\
& =\left(1+a_{1}^{B}\right)^{2} \operatorname{Var}_{t}\left(\int_{t}^{s} r(u) \mathrm{d} u\right)+\left(a_{1}^{A}\right)^{2} \operatorname{Var}_{t}\left(\int_{t}^{u} r(v) \mathrm{d} v\right)+2 a_{1}^{A}\left(1+a_{1}^{B}\right) E_{t}^{Q}\left[\int_{t}^{s} b(v, s) \mathrm{d} Z_{r}(v) \times \int_{t}^{u} b(v, u) \mathrm{d} Z_{r}(v)\right] \\
& =\left(1+a_{1}^{B}\right)^{2} \operatorname{Var}_{t}\left(\int_{t}^{s} r(u) \mathrm{d} u\right)+\left(a_{1}^{A}\right)^{2} \operatorname{Var}_{t}\left(\int_{t}^{u} r(v) \mathrm{d} v\right)+2 a_{1}^{A}\left(1+a_{1}^{B}\right) \int_{t}^{u} \rho(v, s) b(v, u) \mathrm{d} v .
\end{aligned}
$$


Thus, combining (34)-(38), we deduce the pricing formula of the loan issued by secondary firm $B$.

\section{The Pricing of Loan CDS}

This paper assumes that the contract will terminate when the borrower defaults or prepays the loan in advance. We consider a simple situation that $\tau_{p}$ and $\tau_{d}$ are independent. Therefore,

$$
\begin{gathered}
P_{t}\left(\tau_{d}>x, \tau_{p}>y\right)=E_{t}^{Q}\left[\mathrm{e}^{-\int_{t}^{x} \lambda(u) \mathrm{d} u-\int_{t}^{y} \xi(u) \mathrm{d} u}\right], \\
\frac{\mathrm{d} P_{t}(\tau>s)}{\mathrm{d} s}=-(\lambda(s)+\xi(s)) E_{t}^{Q}\left[\mathrm{e}^{-\int_{t}^{s}(\lambda(u)+\xi(u)) \mathrm{d} u}\right], \\
\frac{\partial P_{t}\left(\tau_{d}>x, \tau_{p}>y\right)}{\partial x \partial y}=E_{t}^{Q}\left[\lambda(x) \xi(y) \mathrm{e}^{-\int_{t}^{x} \lambda(u) \mathrm{d} u-\int_{t}^{y} \xi(u) \mathrm{d} u}\right],
\end{gathered}
$$

where $\tau=\tau_{d} \wedge \tau_{p}$.

Firm $C$ has a loan from firm $J$ with the maturity date $T_{1}$. The loan satisfies the above conditions. To seek protection against the possible loss, firm $C$ buys a credit default swap with the maturity date $T_{2}\left(T_{2}<T_{1}\right)$ from firm $K$ on condition that firm $C$ gives the payments to seller $K$ at a fixed swap rate $c$ in time while seller $K$ promises to compensate buyer $C$ for the loss caused by the default of firm $J$ at a certain rate $R$ ( $R$ is the recovery rate of the loan). Each party has the obligation to make payments until its own default. The source of credit risk may be from three parties: the borrower of the loan, the buyer of CDS and the seller of CDS. In the following, we discuss a simple situation which only contains the default risk from reference firm $J$ and the CDS's seller $K$.

The default intensity and the intensity of repaying the loan in advance of firm $J$ satisfy the following equations

$$
\lambda^{J}(t)=a_{0}^{J}+a_{1}^{J} r(t), \xi(t)=b_{0}^{J} .
$$

The default intensity and the intensity of repaying the loan in advance of firm $K$ satisfy the following equations

$$
\lambda^{K}(t)=a_{0}^{K}+a_{1}^{K} r(t)+I_{\left\{\tau_{d}^{J}<t\right\}} \frac{a_{2}^{K}}{a_{3}^{K}\left(t-\tau_{d}^{J}\right)+1}, \xi^{K}(t)=b_{0}^{K} .
$$

Firstly, the time- 0 market value of buyer $C$ 's payments to seller $K$ is

$$
P V(t)=c E_{t}^{Q}\left(\int_{t}^{T_{2}} \mathrm{e}^{-\int_{t^{s}}^{s}(u) \mathrm{d} u} I_{\left\{\tau^{J}>s\right\}} \mathrm{d} s\right)=c E_{t}^{Q}\left(\int_{t}^{T_{2}} \mathrm{e}^{-\int_{t}^{s}\left(r(u)+\lambda^{J}(u)+\xi^{J}(u)\right) \mathrm{d} u} \mathrm{~d} s\right) .
$$

Secondly, the time- 0 market value of seller $K$ 's promised payoff in case of firm $J$ 's default is

$$
\begin{aligned}
& P V_{\text {loss }}=E^{Q}\left[\mathrm{e}^{\int_{0}^{\tau_{d}^{J}} r(u) \mathrm{d} u}\left(1-R\left(\tau_{d}^{J}\right)\right) M\left(\tau_{d}^{J}\right) I_{\left\{\tau_{d}^{J}<T_{2}\right\}} I_{\left\{\tau_{d}^{K}>T_{2}\right\}}\right] \\
& =E^{Q}\left[\mathrm{e}^{\int_{0}^{\tau_{d}^{J}} r(u) \mathrm{d} u}\left(1-R\left(\tau_{d}^{J}\right)\right) M\left(\tau_{d}^{J}\right) I_{\left\{\tau_{d}^{J}<T_{2}\right\}} E^{Q}\left(I_{\left\{\tau_{d}^{K}>T_{2}\right\}} \mid \mathcal{G}_{0}^{K}\right)\right] \\
& =E^{Q}\left[\mathrm{e}^{\int_{0}^{\int_{d}^{J}} r(u) \mathrm{d} u}\left(1-R\left(\tau_{d}^{J}\right)\right) M\left(\tau_{d}^{J}\right) I_{\left\{\tau_{d}^{J}<\tau_{2}\right\}} \mathrm{e}^{-\int_{0}^{T_{2}} \lambda^{K}(u) \mathrm{d} u}\right] \\
& =E^{Q}\left[\mathrm{e}^{\int_{0}^{\tau_{d}^{J}} r(u) \mathrm{d} u}\left(1-R\left(\tau_{d}^{J}\right)\right) M\left(\tau_{d}^{J}\right) I_{\left\{\tau_{d}^{J}<T_{2}\right\}} \mathrm{e}^{-\int_{0}^{T_{2}}\left(a_{0}^{K}+a_{1}^{K} r(u)+I_{\left\{\tau_{d}^{J}<u\right\}} \frac{a_{2}^{K}}{a_{3}^{K}\left(u-\tau_{d}^{J}\right)+1}\right)} \mathrm{d} u\right] .
\end{aligned}
$$

Let $-a_{2}^{K}=a_{3}^{K}=C^{A}>0$, then 


$$
\begin{aligned}
& E^{Q}\left[\mathrm{e}^{-\int_{0}^{\tau_{d}^{J}} r(u) \mathrm{d} u}\left(1-R\left(\tau_{d}^{J}\right)\right) M\left(\tau_{d}^{J}\right) I_{\left\{\tau_{d}^{J}<T_{2}\right\}} \mathrm{e}^{-\int_{0}^{T_{2}}\left(a_{0}^{K}+a_{1}^{K} r(u)+I_{\left\{\tau_{d}^{J}<u\right\}} \frac{a_{2}^{K}}{a_{3}^{K}\left(u-\tau_{d}^{J}\right)+1}\right) \mathrm{d} u}\right] \\
& =E^{Q}\left[\mathrm { e } ^ { - \int _ { 0 } ^ { \tau _ { d } ^ { J } } r ( u ) \mathrm { d } u } \left(1-R\left(\tau_{d}^{J}\right) M\left(\tau_{d}^{J}\right) I_{\left\{\tau_{d}^{J}<T_{2}\right\}} \mathrm{e}^{-\int_{0}^{T_{2}}\left(a_{0}^{K}+a_{1}^{K} r(u)+I_{\left\{\tau_{d}^{J}<u\right\}} \frac{-C^{A}}{C^{A}\left(u-\tau_{d}^{J}\right)+1}\right)} \mathrm{d} u\right.\right.
\end{aligned}
$$

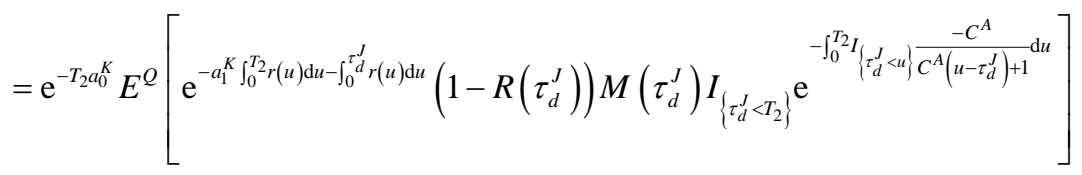

$$
\begin{aligned}
& =\mathrm{e}^{-T_{2} a_{0}^{K}} E^{Q}\left[\mathrm{e}^{-a_{1}^{K} \int_{0}^{T_{2}} r(u) \mathrm{d} u-\int_{0}^{\tau_{d}^{J}} r(u) \mathrm{d} u}\left(1-R\left(\tau_{d}^{J}\right)\right) M\left(\tau_{d}^{J}\right) I_{\left\{\tau_{d}^{J}<T_{2}\right\}}\left(C^{A}\left(T^{2}-\tau_{d}^{J}\right)\right)+1\right] \\
& =\mathrm{e}^{-T_{2} a_{0}^{K}} E^{Q}\left[\int_{0}^{T_{2}} \mathrm{e}^{-a_{1}^{K} \int_{0}^{T_{2}} r(u) \mathrm{d} u}(1-R(s)) M(s) \lambda^{J}(s) \mathrm{e}^{-\int_{0}^{\tau_{d}^{J}}\left(r(u)+\lambda^{J}(u)+\xi^{J}(u)\right) \mathrm{d} u}\left(C^{A}\left(T_{2}-s\right)+1\right) \mathrm{d} s\right] \\
& =\mathrm{e}^{-T_{2} a_{0}^{K}} \int_{0}^{T_{2}}(1-R(s)) M(s)\left(C^{A}\left(T_{2}-s\right)+1\right) E^{Q}\left[\lambda^{J}(s) \mathrm{e}^{-a_{1}^{K} \int_{0}^{T_{2}} r(u) \mathrm{d} u} \mathrm{e}^{-\int_{0}^{s}\left(r(u)+\lambda^{J}(u)+\xi^{J}(u)\right) \mathrm{d} u}\right] \mathrm{d} s .
\end{aligned}
$$

Now, we calculate

$$
\begin{aligned}
& E^{Q}\left[\lambda^{J}(s) \mathrm{e}^{-a_{1}^{K} \int_{0}^{T_{2} r(u) d u}} \mathrm{e}^{-\int_{0}^{s}\left(r(u)+\lambda^{J}(u)+\xi^{J}(u)\right) \mathrm{d} u}\right]
\end{aligned}
$$

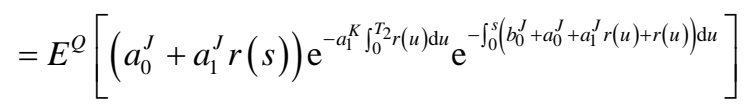

$$
\begin{aligned}
& =\mathrm{e}^{-s\left(b_{0}^{J}+a_{0}^{J}\right)} E^{Q}\left[\left(a_{0}^{J}+a_{1}^{J} r(s)\right) \mathrm{e}^{-a_{1}^{K} \int_{0}^{T_{2} r(u) d u}} \mathrm{e}^{-\left(1+a_{1}^{J}\right) \int_{0}^{S} r(u) \mathrm{d} u}\right] \\
& =a_{0}^{J} \mathrm{e}^{-s\left(b_{0}^{J}+a_{0}^{J}\right)} E^{Q}\left[\mathrm{e}^{-a_{1}^{K} \int_{0}^{T_{2}} r(u) \mathrm{d} u} \mathrm{e}^{-\left(1+a_{1}^{J}\right) \int_{0}^{s} r(u) \mathrm{d} u}\right] \\
& +a_{1}^{J} \mathrm{e}^{-s\left(b_{0}^{J}+a_{0}^{J}\right)} E^{Q}\left[r(s) \mathrm{e}^{-a_{1}^{K} \int_{0}^{T_{2}} r(u) \mathrm{d} u} \mathrm{e}^{-\left(1+a_{1}^{J}\right) \int_{0}^{S} r(u) \mathrm{d} u}\right] .
\end{aligned}
$$

Let $X=\int_{0}^{T_{2}} r(u) \mathrm{d} u, Y=\int_{0}^{s} r(u) \mathrm{d} u$. For any normal random variables $X$ and $Y$,

$$
\begin{gathered}
E\left(\mathrm{e}^{A X+B Y}\right)=\exp \left[A \mu_{X}+B \mu_{Y}+\frac{1}{2}\left(A^{2} \sigma_{X}^{2}+2 \sigma_{X Y} A B+B^{2} \sigma_{Y}^{2}\right)\right] \text {. So we have } \\
\mu_{X}=\mu\left(\int_{0}^{T_{2}} r(u) \mathrm{d} u\right)=\int_{0}^{T_{2}} f(0, u) \mathrm{d} u+\int_{0}^{T_{2}} \frac{b(0, u)^{2}}{2} \mathrm{~d} u \\
\mu_{Y}=\mu\left(\int_{0}^{s} r(u) \mathrm{d} u\right)=\int_{0}^{s} f(0, u) \mathrm{d} u+\int_{0}^{s} \frac{b(0, u)^{2}}{2} \mathrm{~d} u . \\
\sigma_{X}^{2}=\operatorname{Var}\left(\int_{0}^{T_{2}} r(u) \mathrm{d} u\right)=E^{Q}\left[\int_{0}^{T_{2}} b\left(u, T_{2}\right) \mathrm{d} Z_{r}(u) \times \int_{0}^{T_{2}} b\left(u, T_{2}\right) \mathrm{d} Z_{r}(u)\right]=\int_{0}^{T_{2}} b\left(u, T_{2}\right)^{2} \mathrm{~d} u . \\
\sigma_{Y}^{2}=\operatorname{Var}\left(\int_{0}^{s} r(u) \mathrm{d} u\right)=E^{Q}\left[\int_{0}^{s} b(u, s) \mathrm{d} Z_{r}(u) \times \int_{0}^{s} b(u, s) \mathrm{d} Z_{r}(u)\right]=\int_{0}^{T_{2}} b(u, s)^{2} \mathrm{~d} u . \\
\sigma_{X Y}=E^{Q}\left[\int_{0}^{T_{2}} r(u) \mathrm{d} u \int_{0}^{s} r(u) \mathrm{d} u\right]-E^{Q}\left[\int_{0}^{T_{2}} r(u) \mathrm{d} u\right] E^{Q}\left[\int_{0}^{s} r(u) \mathrm{d} u\right] \\
=E^{Q}\left[\int_{0}^{T_{2}} b\left(u, T_{2}\right) \mathrm{d} Z_{r}(u) \times \int_{0}^{s} b(u, s) \mathrm{d} Z_{r}(u)\right]=\int_{0}^{s} b\left(u, T_{2}\right) b(u, s) \mathrm{d} u .
\end{gathered}
$$




$$
\left.E^{Q}\left[\mathrm{e}^{-a_{1}^{K} \int_{0}^{T_{2}} r(u) \mathrm{d} u} \mathrm{e}^{-\left(1+a_{1}^{J}\right)}\right) \int_{0^{r}}^{s^{r}(u) \mathrm{d} u}\right]=\exp \left[A \mu_{X}+B \mu_{Y}+\frac{1}{2}\left(A^{2} \sigma_{X}^{2}+2 \sigma_{X Y} A B+B^{2} \sigma_{Y}^{2}\right)\right] .
$$

Then, we substitute (48)-(51) into (52) and get $E^{Q}\left[\mathrm{e}^{-a_{1}^{K} \int_{0}^{T_{2} r} r(u) \mathrm{d} u} \mathrm{e}^{-\left(1+a_{1}^{J}\right) \int_{0}^{s} r(u) \mathrm{d} u}\right]$ Let $A=1, X=-a_{1}^{K} \int_{0}^{T_{2}} r(u) \mathrm{d} u-\left(1+a_{1}^{J}\right) \int_{0}^{s} r(u) \mathrm{d} u ; B=0, Y=r(s)$. We have

$$
\begin{aligned}
& \mu_{X}=\mu\left(-a_{1}^{K} \int_{0}^{T_{2}} r(u) \mathrm{d} u-\left(1+a_{1}^{J}\right) \int_{0}^{s} r(u) \mathrm{d} u\right) \\
&=-a_{1}^{K} \int_{0}^{T_{2}} f(0, u) \mathrm{d} u+\int_{0}^{T_{2}} \frac{b(0, u)^{2}}{2} \mathrm{~d} u-\left(1+a_{1}^{J}\right)\left(\int_{0}^{s} f(0, u) \mathrm{d} u+\int_{0}^{s} \frac{b(0, u)^{2}}{2} \mathrm{~d} u\right) \\
& \mu_{Y}=\mu(r(s))=f(0, s)+\frac{b(0, u)^{2}}{2} . \\
& \sigma_{X}^{2}=\operatorname{Var}\left(-a_{1}^{K} \int_{0}^{T_{2}} r(u) \mathrm{d} u-\left(1+a_{1}^{J}\right) \int_{0}^{s} r(u) \mathrm{d} u\right) \\
&=\left(a_{1}^{K}\right)^{2} \operatorname{Var}\left(\int_{0}^{T_{2}} r(u) \mathrm{d} u\right)+\left(1+a_{1}^{J}\right)^{2} \operatorname{Var}\left(\int_{0}^{s} r(u) \mathrm{d} u\right) \\
& \quad+2 a_{1}^{K}\left(1+a_{1}^{J}\right) \operatorname{Cov}\left(\int_{0}^{T_{2}} r(u) \mathrm{d} u, \int_{0}^{s} r(u) \mathrm{d} u\right) \\
& \sigma_{Y}^{2}=\operatorname{Var}(r(s))=\int_{0}^{s} \rho(v, s)^{2} \mathrm{~d} v . \\
& \sigma_{X Y}= \operatorname{Cov}\left(r(s),-a_{1}^{K} \int_{0}^{T_{2}} r(u) \mathrm{d} u-\left(1+a_{1}^{J}\right) \int_{0}^{s} r(u) \mathrm{d} u\right) \\
&=-a_{1}^{K} \operatorname{Cov}\left(r(s), \int_{0}^{T_{2}} r(u) \mathrm{d} u\right)-\left(1+a_{1}^{J}\right) \operatorname{Cov}\left(r(s), \int_{0}^{s} r(u) \mathrm{d} u\right) \\
&=-a_{1}^{K}\left(E^{Q}\left[r(s) \int_{0}^{T_{2}} r(u) \mathrm{d} u\right]-E^{Q}[r(s)] E^{Q}\left[\int_{0}^{T_{2}} r(u) \mathrm{d} u\right]\right) \\
&-\left(1+a_{1}^{J}\right)\left(E^{Q}\left[r(s) \int_{0}^{s} r(u) \mathrm{d} u\right]-E^{Q}[r(s)] E^{Q}\left[\int_{0}^{s} r(u) \mathrm{d} u\right]\right) \\
&=-a_{1}^{K} E^{Q}\left[\int_{0}^{s} \rho(v, s) \mathrm{d} Z_{r}(v) \times \int_{0}^{T_{2}} b\left(v, T_{2}\right) \mathrm{d} Z_{r}(v)\right] \\
&-\left(1+a_{1}^{J}\right) E^{Q}\left[\int_{0}^{s} \rho(v, s) \mathrm{d} Z_{r}(v) \times \int_{0}^{s} b(v, s) \mathrm{d} Z_{r}(v)\right] \\
&=-a_{1}^{K} \int_{0}^{s} \rho(v, s) b\left(v, T_{2}\right) \mathrm{d} v-\left(1+a_{1}^{J}\right) \int_{0}^{s} \rho(v, s) b(v, s) \mathrm{d} v .
\end{aligned}
$$

For any normal random variables $X$ and $Y$,

$$
\begin{aligned}
& \frac{\partial E\left[\mathrm{e}^{A X+B Y}\right]}{\partial B}=E\left[B \mathrm{e}^{A X+B Y}\right] \\
& =\exp \left[A \mu_{X}+B \mu_{Y}+\frac{1}{2}\left(A^{2} \sigma_{X}^{2}+2 A B \sigma_{X Y}+B^{2} \sigma_{Y}^{2}\right)\right]\left(\mu_{Y}+B \sigma_{Y}^{2}+A \sigma_{X Y}\right) .
\end{aligned}
$$

Therefore,

$$
\begin{aligned}
& E^{Q}\left[r(s) \mathrm{e}^{\left.-a_{1}^{K} \int_{0}^{T_{2} r(u) \mathrm{d} u} \mathrm{e}^{-\left(1+a_{1}^{J}\right) \int_{0}^{S_{0}} r(u) \mathrm{d} u}\right]}\right. \\
& =\left.\frac{\partial E_{t}^{Q}\left[\mathrm{e}^{-\left(1+a_{1}^{K}\right) \int_{t}^{s} r(u) \mathrm{d} u+B r(s)-a_{1}^{J} \int_{t}^{u} r(v) \mathrm{d} v}\right]}{\partial B}\right|_{B=0}=\exp \left(A \mu_{X}+\frac{1}{2} A^{2} \sigma_{X}^{2}\right)\left(\mu_{Y}+A \sigma_{X Y}\right) .
\end{aligned}
$$


We substitute (53)-(55) into(57) and get the above expectation.

Thus, in accordance with the arbitrage-free principle, we obtain the swap rate of loan CDS

$$
c=\frac{P V_{\text {loss }}}{P V(0)}=\frac{E^{Q}\left[\mathrm{e}^{\int_{0}^{\int_{0}^{J}} r(u) \mathrm{d} u}\left(1-R\left(\tau_{d}^{J}\right)\right) M\left(\tau_{d}^{J}\right) I_{\left\{\tau_{d}^{J}<T_{2}\right\}} I_{\left\{\tau_{d}^{K}>T_{2}\right\}}\right]}{E^{Q}\left(\int_{0}^{T_{2}} \mathrm{e}^{-\int_{t}^{S}\left(r(u)+\lambda^{J}(u)+\xi^{J}(u)\right) \mathrm{d} u} \mathrm{~d} s\right)} .
$$

\section{Conclusion}

CDS is one of the credit derivatives with large trading volume in the global financial market. In fact, there is a certain relationship among most of the companies in real market, such as the problem of holding each other's bonds and so on. If a company defaults, it will affect the default possibility of another company. Default contagion is a common phenomenon in financial markets. This paper studies the pricing of CDS with the loan as the reference asset when contagious risk has the attenuation effect. We consider that the default intensity is correlated with the counterparty's default and the interest rate following Vasicek model. The conclusions in this paper can provide the theoretical preparation and suggestions for the credit products development and the research in China. In fact, we only discussed the simple situation. The default of a firm and the prepayment of the loan issued by the firm are independent. Moreover, we price the loan and CDS under the primary-secondary framework. We can also consider other more complex cases, such as the correlation of the default intensity and the prepayment intensity, the looping default effect and so on.

\section{Acknowledgements}

We thank the editor and the referee for their comments. The research is funded by the National Natural Science Foundation of China (No: 11271259) and Funding scheme for training young teachers in Shanghai Colleges (ZZshjr12010). This support is greatly appreciated.

\section{References}

[1] Black, F. and Scholes, M. (1973) The Pricing of Options and Corporate Liabilities. Journal of Political Economy, 81, 637-654. http://dx.doi.org/10.1086/260062

[2] Merton, R.C. (1974) On the Pricing of Corporate Debt: The Risk Structure of Interest Rates. Journal of Finance, 29, 449-470. http://dx.doi.org/10.1111/j.1540-6261.1974.tb03058.x

[3] Duffie, D. and Singleton, K.J. (1995) Modeling Term Structures of Defaultable Bonds. Working Paper, Stanford University Business School.

[4] Jarrow, R.A., Lnado, D. and Turnbull, S. (1994) A Markov Model for the Term Structure of Credit Risk Spreads. Working Paper, Conell University.

[5] Jarrow, R.A. and Turnbull, S. (1995) Pricing Derivatives on Financial Securities Subject to Credit Risk. Journal of Finance, 50, 53-86. http://dx.doi.org/10.1111/j.1540-6261.1995.tb05167.x

[6] Davis, M. and Lo, V. (2001) Infectious Defaults. Quantitative Finance, 1, 382-387. http://dx.doi.org/10.1080/713665832

[7] Jarrow, R.A. and Yu, F. (2001) Counterparty Risk and the Pricing of Defaultable Securities. Journal of Finance, 56, 1765-1799. http://dx.doi.org/10.1111/0022-1082.00389

[8] Bai, Y.F., Hu, X.H. and Ye, Z.X. (2007) A Model for Dependent Default with Hyperbolic Attenuation Effect and Valuation of Credit Default Swap. Applied Mathematics and Mechanics (English Edition), 28, 1643-1649. http://dx.doi.org/10.1007/s10483-007-1211-9

[9] Hao, R.L. and Ye, Z.X. (2011) The Intensity Model for Pricing Credit Securities with Jump-Diffusion and Counterparty Risk. Mathematical Problems in Engineering, 10, 1-16. http://dx.doi.org/10.1155/2011/412565

[10] Hao, R.L., Liu, Y.H. and Wang, S.B. (2014) Pricing Credit Default Swap under Fractional Vasicek Interest Rate Model. Journal of Mathematical Finance, 4, 10-20. http://dx.doi.org/10.4236/jmf.2014.41002

[11] Gu, W.J., Liu, Y.L. and Hao, R.L. (2016) Attenuated Model of Pricing Credit Default Swap under the Fractional Brownian Motion Environment. Journal of Mathematical Finance, 6, 247-259. http://dx.doi.org/10.4236/jmf.2016.62021 
[12] Wu, S. and Liang, J. (2011) Valuation of Mortgage Loan CDS. Applied Mathematics: A Journal of Chinese Universities, 26, 269-278.

[13] Zhang, X.Y. (2012) The Pricing of Credit Default Swap Based on Project Loans. The Dissertation of Nanjing University of Science and Technology, Nanjing.

[14] Howard, S., Shunichiro, U. and Zhen, W. (2007) Valuation of loan CDS and CDX. Working Paper, Stanford University.

[15] Zhen, W. (2007) Valuation of Loan CDS under Intensity Based Model. Working Paper, Stanford University.

[16] Hao, R.L., Zhang, J.Q., Liu, Y.H. and Hu, Z.H. (2016) Pricing Credit Default Swap with Contagious Risk and Simulation. Shanghai Jiaotong University (Science), 21, 57-62. 


\section{Appendix}

\section{Proof of Lemma 1}

Proof. Firstly, from Section 2, we have

$$
\begin{aligned}
P\left(\tau_{d}^{i}>T \mid \mathcal{G}_{t}^{i}\right) & =E\left[I_{\left\{\tau_{d}^{i}>T\right\}} \mid \mathcal{G}_{t}^{i}\right]=P\left(\tau_{d}^{i}>T \mid \mathcal{G}_{0}^{i} \vee \mathcal{F}_{t}^{i}\right)=I_{\left\{\tau_{d}^{i}>t\right\}} \frac{P\left(\tau_{d}^{i}>T \mid \mathcal{G}_{0}^{i}\right)}{P\left(\tau_{d}^{i}>t \mid \mathcal{G}_{0}^{i}\right)} \\
& =I_{\left\{\tau_{d}^{i}>t\right\}} \frac{\exp \left(-\int_{0}^{T} \lambda^{i}(u) \mathrm{d} u\right)}{\exp \left(-\int_{0}^{t} \lambda^{i}(u) \mathrm{d} u\right)}=I_{\left\{\tau_{d}^{i}>t\right\}} \exp \left(-\int_{t}^{T} \lambda^{i}(u) \mathrm{d} u\right) .
\end{aligned}
$$

Similarly, we have

$$
\begin{aligned}
P\left(\tau_{p}^{i}>T \mid \mathcal{G}_{t}^{i}\right) & =E\left[I_{\left\{\tau_{p}^{i}>T\right\}} \mid \mathcal{G}_{t}^{i}\right]=P\left(\tau_{p}^{i}>T \mid \mathcal{G}_{0}^{i} \vee \mathcal{H}_{t}^{i}\right)=I_{\left\{\tau_{p}^{i}>t\right\}} \frac{P\left(\tau_{p}^{i}>T \mid \mathcal{G}_{0}^{i}\right)}{P\left(\tau_{p}^{i}>t \mid \mathcal{G}_{0}^{i}\right)} \\
& =I_{\left\{\tau_{p}^{i}>t\right\}} \frac{\exp \left(-\int_{0}^{T} \xi^{i}(u) \mathrm{d} u\right)}{\exp \left(-\int_{0}^{t} \xi^{i}(u) \mathrm{d} u\right)}=I_{\left\{\tau_{p}^{i}>t\right\}} \exp \left(-\int_{t}^{T} \xi^{i}(u) \mathrm{d} u\right) .
\end{aligned}
$$

\section{Proof of Theorem 1}

Proof. Firstly, $E_{t}^{Q}\left[M\left(\tau_{p}^{i}\right) \mathrm{e}^{-\int_{t}^{\tau_{t}^{i}} r(u) \mathrm{d} u} I_{\left\{\tau_{p}^{i}<T\right\}}\right]=E_{t}^{Q}\left[\int_{t}^{T} M(s) \xi^{i}(s) \mathrm{e}^{-\int_{t}^{s}\left(r(u)+\xi^{i}(u)+\lambda^{i}(u)\right) \mathrm{d} u} \mathrm{~d} s\right]$.

$$
\begin{aligned}
& E_{t}^{Q}\left[M\left(\tau_{p}^{i}\right) \mathrm{e}^{-\int_{t}^{t_{p}^{i}} r(u) \mathrm{d} u} I_{\left\{\tau_{p}^{i}<T\right\}}\right]=E_{t}^{Q}\left(M\left(\tau_{p}^{i}\right) \mathrm{e}^{-\int_{t}^{\tau_{t}^{i}} r(u) \mathrm{d} u} I_{\left\{\tau_{p}^{i}<T\right\}} \mid \mathcal{G}_{t}^{i}\right) \\
& =E_{t}^{Q}\left[\int_{t}^{T} M(y) \mathrm{e}^{-\int_{t}^{y} r(u) \mathrm{d} u} \xi^{i}(y) \mathrm{e}^{-\int_{t}^{y} \xi^{i}(u) \mathrm{d} u} \mathrm{~d} y \int_{y}^{\infty} \lambda^{i}(x) \mathrm{e}^{-\int_{t}^{x} \lambda^{i}(u) \mathrm{d} u} \mathrm{~d} x\right] \\
& =E_{t}^{Q}\left[\int_{t}^{T} M(s) \xi^{i}(s) \mathrm{e}^{-\int_{t}^{s}\left(r(u)+\xi^{i}(u)+\lambda^{i}(u)\right) \mathrm{d} u} \mathrm{~d} s\right] .
\end{aligned}
$$

Secondly,

$$
\begin{aligned}
& E_{t}^{Q}\left[M\left(\tau_{d}^{i}\right) R\left(\tau_{d}^{i}\right) \mathrm{e}^{-\int_{t}^{\tau_{t}^{i}} r(u) \mathrm{d} u} I_{\left\{\tau_{d}^{i}<T\right\}}\right]=E_{t}^{Q}\left(M\left(\tau_{d}^{i}\right) R\left(\tau_{d}^{i}\right) \mathrm{e}^{-\int_{t}^{\tau_{t}^{i}} r(u) \mathrm{d} u} I_{\left\{\tau_{d}^{i}<T\right\}} \mid \mathcal{G}_{t}^{i}\right) \\
& =E_{t}^{Q}\left[\int_{t}^{T} M(x) R(x) \mathrm{e}^{-\int_{t}^{x} r(u) \mathrm{d} u} \lambda^{i}(x) \mathrm{e}^{-\int_{t}^{x} \lambda^{i}(u) \mathrm{d} u} \mathrm{~d} x \int_{x}^{\infty} \xi^{i}(y) \mathrm{e}^{-\int_{t}^{y} \lambda^{i}(u) \mathrm{d} u} \mathrm{~d} y\right] \\
& =E_{t}^{Q}\left[\int_{t}^{T} R(s) M(s) \lambda^{i}(s) \mathrm{e}^{-\int_{t}^{s}\left(r(u)+\xi^{i}(u)+\lambda^{i}(u)\right) \mathrm{d} u} \mathrm{~d} s\right] .
\end{aligned}
$$

At last,

$$
\begin{aligned}
& E_{t}^{Q}\left[\int_{t}^{T} Y \mathrm{e}^{-\int_{t}^{s} r(u) \mathrm{d} u} I_{\left\{\tau^{i}>s\right\}} \mathrm{d} s\right]=Y E_{t}^{Q}\left(\int_{t}^{T} \mathrm{e}^{-\int_{t}^{S} r(u) \mathrm{d} u} I_{\left\{\tau_{d}^{i}>s\right\}} I_{\left\{\tau_{p}^{i}>s\right\}} \mathrm{d} s \mid \mathcal{G}_{t}^{i}\right) \\
& =L E_{t}^{Q}\left[\int_{s}^{\infty} \mathrm{d} x \int_{s}^{\infty} \int_{t}^{T} \mathrm{e}^{-\int_{t}^{s} r(u) \mathrm{d} u} \mathrm{~d} s \lambda^{i}(x) \mathrm{e}^{-\int_{t}^{x^{i}} \lambda^{i}(u) \mathrm{d} u} \xi^{i}(y) \mathrm{e}^{-\int_{t}^{y} \lambda^{i}(u) \mathrm{d} u} \mathrm{~d} y\right]
\end{aligned}
$$

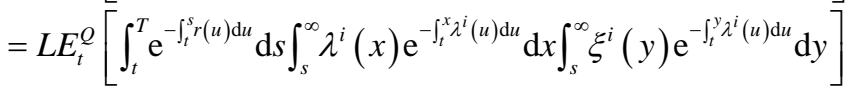

$$
\begin{aligned}
& =L E_{t}^{Q}\left[\int_{t}^{T} \mathrm{e}^{-\int_{t}^{s}\left(r(u)+\xi^{i}(u)+\lambda^{i}(u)\right) \mathrm{d} u} \mathrm{~d} s\right] .
\end{aligned}
$$

Therefore, the theorem is deduced. 


\section{Submit or recommend next manuscript to SCIRP and we will provide best service for you:}

Accepting pre-submission inquiries through Email, Facebook, LinkedIn, Twitter, etc.

A wide selection of journals (inclusive of 9 subjects, more than 200 journals)

Providing 24-hour high-quality service

User-friendly online submission system

Fair and swift peer-review system

Efficient typesetting and proofreading procedure

Display of the result of downloads and visits, as well as the number of cited articles

Maximum dissemination of your research work

Submit your manuscript at: http://papersubmission.scirp.org/ 